\title{
Il futuro di tutto
}

\section{Marco Lombardi}

Editor in Chief, Giornale di Clinica Nefrologica e Dialisi
Come Editor del GCND ho deciso di pubblicare un manifesto dedicato alla responsabilizzazione dei medici nefrologi e delle loro società scientifiche per la difesa della nostra Casa Comune. C'è bisogno di aiutare il nostro pianeta per avere un futuro noi tutti e quindi anche i nostri pazienti.

Sensibile a questa problematica, non appena l'amico nefrologo Franco Bergesio mi ha proposto la cosa ho immediatamente aderito, perché qualsiasi cosa facciamo è sempre troppo poco, e non capisco come troppa gente non se ne renda conto.

Quindi pubblicare in ambo le lingue questo manifesto sul GCND è il minimo che possa fare. Spiegavo a Franco che il GCND viene letto anche da pazienti, infermieri, dietisti, ecc., e questo è un plusvalore perché l'unico vero scopo di questo documento è diffondere il "verbo" di più e il prima possibile: ognuno faccia la sua piccola parte, non solo nella propria vita quotidiana fuori dall'ospedale, ma anche e proprio all'interno di ospedali, ambulatori, case di cura, e ovunque possibile.

Il problema è enorme ed interesserà tutti indistintamente. Ovviamente, è principalmente politico, ma ognuno di noi dovrebbe prendersi la propria responsabilità per capire che se quest'era definita antropogenica (1) sarà ricordata ... (da chi?) significa che in un tempo eccezionalmente breve l'essere umano sta bruciando la sua Casa senza preoccuparsene o preoccupandosene troppo superficialmente. Questo nasconde un efferato egoismo a cui fanno pendant razzismo, consumismo ... ed è meglio che mi fermi. Troppo pochi - se pensiamo a quanti siamo ormai - si salvano da questi bassi sentimenti, ma se resteranno così pochi non si salveranno, paradossalmente, neppure loro.

Il problema è già all'attenzione della classe dei medici nefrologi, almeno di quelli che hanno acquisito la consapevolezza della necessità di acquisire una nuova etica, come scrive Bianca Covella proprio sul Giornale della nostra Società (2) ove riporta anche del recente Global Meeting sulla Green Nephrology, organizzato nell'ambito del congresso della ASN nel 2018 in collaborazione con la ISN. Infatti, come afferma

Received: January 03, 2020

Accepted: January 07, 2020

Published online: March 05, 2020

Indirizzo per la corrispondenza:

Marco Lombardi

Ospedale del Mugello

Via Della Resistenza, 60

50032 Borgo San Lorenzo, Firenze, Italia

lombardim@tin.it
Bianca (2), la nostra professione, quella sanitaria, oltre a possedere la capacità di proteggere e difendere la salute pubblica, ne deve avere anche la responsabilità e pertanto deve agire. Come? Rendendo edotti i cittadini, gli amministratori e, perché no, i politici sui pericoli, i rischi e le possibili opportunità di difesa e prevenzione dei cambiamenti climatici legati all'inquinamento della nostra Casa, garantendo i cambiamenti necessari per il benessere umano (3) e di conseguenza di tutte le altre specie viventi, animali e vegetali, senza dimenticare tutti gli altri elementi (suolo, acque, ecc).

Come ha affermato lo scrittore Jonathan Franzen sul "The New Yorker", riportato anche da Internazionale (4), quanto sta accadendo potrà portare $\mathrm{a}$ implosioni economiche anche nei Paesi più abbienti, a situazioni apocalittiche e migrazioni cosi importanti, che quelle che attualmente ci spaventano potrebbero essere irrisorie. Sempre secondo J.F., si dovrebbe cominciare a ripensare al significato della parola SPERANZA ... concetto difficile se si pensa che negli ultimi 30 anni abbiamo immesso una quantità di $\mathrm{CO} 2$ pari a quella immessa nei precedenti duecento anni di industrializzazione. Concetto difficile se, come scrive Franzen, lo scelleratismo delle posizioni Repubblicane negli USA è folle, ma il negazionismo è purtroppo radicato anche nei progressisti. L'obiettivo dovrebbe essere zero emissioni, globalmente nei prossimi tre decenni ... e se anche le azioni di un singolo individuo non hanno alcun effetto sul clima, ciò non significa che siano insignificanti ... (4).

L'esortazione del documento che presento è dunque anche un'esortazione per una "economia civile e solidale" dove dovrebbe prevalere il bene comune sul profitto dei singoli; è un'esortazione a cercare di non ridurre ulteriormente la resilienza della nostra Casa Comune prendendo coscienza anche nel mondo nefrologico della problematica ... perché in fondo si tratta di curare anche il nostro pianeta oltre che $i$ nostri pazienti.

\section{Bibliografia}

1. Baichwal J, De Pencier N, Burtynsky E. Antropocene: L'epoca umana. Film distribuito da Stensen.

2. Covella B, Rossi L, Lisi P, Corciulo S, Manno E, Lomonte C. II nefrologo di fronte ai cambiamenti climatici. G Ital Nefrol 2019;36(6):1-5.

3. Watts N, Adger WN, Ayeb-Karlsson S, Bai Y, Byass P, et al. The Lancet Countdown: tracking progress on health and climate change. Lancet 2017;389(10074):1151-64.

4. Franzen J. L'ottimismo fa male? Smettiamo difingere. Internazionale. 2019;1329:46-50. 